\title{
Analysis of slice transverse emittance evolution in a very-high-frequency gun photoinjector
}

\author{
Han Chen, Lianmin Zheng®, Pengwei Huang, Chuangye Song, Yingchao Du๑," Renkai Li, \\ Wenhui Huang, and Chuanxiang Tang \\ Accelerator Laboratory, Department of Engineering Physics, Tsinghua University, Beijing 100084, China
}

(Received 13 September 2021; accepted 6 December 2021; published 21 December 2021)

\begin{abstract}
Emittance is a key figure-of-merit for high brightness electron beams that enable the success of a broad range of modern accelerator-based instruments. With the successful implementation of the emittance compensation scheme and beam-shaping techniques to align each temporal beam slice in phase space, slice emittance starts to play a more dominating role in determining the beam quality. In this paper, we develop an approach, combining simulations and analytical calculations, to systematically study the evolution of the slice emittance in a very high frequency gun photoinjector, which is under intense R\&D worldwide for driving the next generation continuous-wave accelerators. Our approach is capable of tracing various sources that introduce curvature in the transverse phase space, corresponding to the growth of the slice emittance. It is shown that nonlinear transverse space charge forces in the vicinity of cathodes and the spherical aberration of the focusing solenoid are the main sources of nonlinear forces, while in a long drift section space charge forces can actually compensate for nonlinear transverse position-momentum correlations and hence recover the emittance. We further demonstrate that the sources of nonlinearities can be controlled by tailoring the transverse beam density profile and the design of solenoid lenses.
\end{abstract}

DOI: $10.1103 /$ PhysRevAccelBeams.24.124402

\section{INTRODUCTION}

Beam transverse emittance is a key figure of merit for photoinjector-driven electron accelerators, such as freeelectron lasers (FELs) [1-6], inverse Compton scattering sources $[7,8]$, energy recovery linacs $[9,10]$, and ultrafast electron diffraction [11,12] and microscopy [13-15]. The ultimate performance of these applications is essentially determined by the lowest transverse emittance of the electron beam achieved in the photoinjectors.

In the past decades, frontier scientific researches desire electron beams with not only low emittance but also highrepetition rates. For example, x-ray FEL and ultrafast electron diffraction or microscopy facilities operating at $\mathrm{MHz}-\mathrm{class}$ repetition rates are proposed and rapidly developing to satisfy new science demands. To produce the electron beams with $\mathrm{MHz}$-class repetition rates, several types of continuous-wave $(\mathrm{CW})$ photocathode guns have been developed, such as dc guns [16,17], superconducting rf guns [18], and very high frequency (VHF) rf guns [19]. The VHF gun has been selected as the electron source in

*dych@mail.tsinghua.edu.cn

Published by the American Physical Society under the terms of the Creative Commons Attribution 4.0 International license. Further distribution of this work must maintain attribution to the author(s) and the published article's title, journal citation, and DOI. several influential user facilities [20,21] because it can achieve high-cathode gradient and has high-reliability meanwhile.

The optimizations of the transverse emittance for relatively low-repetition rate (100 Hz class) beams in S- or Lband photoinjectors have been reported in numerous works [22-24], and low-transverse emittance has been achieved in these photoinjectors. However, the beam dynamics analysis in VHF gun photoinjectors has not been studied sufficiently, and some new features about the evolution of the beam emittance require further exploration. Compared with the S-band or L-band guns [25-27], the VHF gun is operated with lower frequency, i.e., longer wavelength, thus the initial laser duration can be long to suppress the space charge (SC) force and the initial laser spot size can be small to reduce the initial thermal emittance. Moreover, the VHF gun has lower cathode gradient than the S-band or Lband guns, thus the expansion of transverse beam size in the beamline is large and the influence of solenoid spherical aberration on the emittance growth is relatively severe.

This paper focuses on the beam transverse emittance evolution in a VHF gun photoinjector. If an electron bunch is divided into thin temporal slices which are not mutually interacting, the emittance of each slice is called slice emittance, and the emittance of the sum of all slices is called projected emittance. Different slices experience different focusing effects due to time-dependent acceleration fields and SC forces, leading to different angles of 
VHF GUN

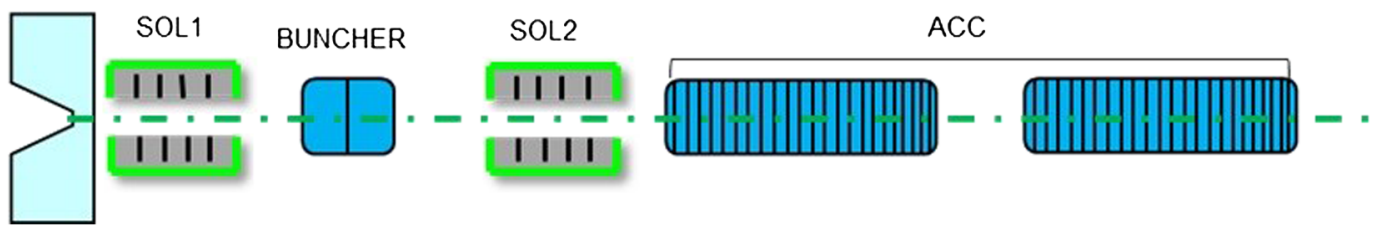

FIG. 1. Sketch of a VHF gun photoinjector designed by Tsinghua University.

inclination of the slices in the transverse phase space. The misalignment of different temporal slices in the transverse phase space results in a growth of the projected emittance. The projected emittance can be orders of magnitude larger than the slice emittance in photoinjectors. In the past decades, numerous theoretical models have been established and experiments have been performed to best align the transverse phase space of the beam temporal slices, thus minimizing the projected emittance of the entire beam. Until now, simulations and experiments reveal that the projected emittance after optimization is very close to the slice emittance, and the slice emittance is becoming a bottleneck limiting the further emittance reduction. Besides, FEL's performance greatly depends on the beam slice emittance in a slippage length [28]. Therefore, it is significant to understand and control the growth of the slice emittance in photoinjectors. The slice emittance is the quadrature sum of the thermal emittance and the emittance growth due to various nonlinear radial forces. In this paper, the evolution of the slice transverse emittance is analyzed in a VHF gun photoinjector, and the sources of nonlinear transverse forces leading to slice emittance growth are studied with theoretical analysis and beam dynamics simulations. Furthermore, we will demonstrate that the nonlinear transverse forces can be reduced by tailoring the transverse beam density profile and the dimensions of the solenoids. We will also demonstrate that in a long drift section the nonlinear SC forces can compensate for the nonlinear transverse position-momentum correlations and hence recover the emittance. The slice emittance compensation will be explained by analyzing the evolution of the beam charge transverse distribution. It should be noted that the methodology employed in this paper to analyze the slice emittance evolution in the VHF gun photoinjector is also valuable to other conventional injectors, such as $\mathrm{S}$ - or L-band photoinjectors.

The paper is organized as follows. Section II gives the relation between the projected emittance and the slice emittance based on simulations of a VHF gun photoinjector. In Sec. III, we analyze the evolution of the slice emittance in different sections of the photoinjector, including the photo-gun, the solenoids, the drift, the buncher, and the linac boosters. Finally in Sec. IV, we will demonstrate that the evolution analysis exhibited in the VHF gun photoinjector can also be employed in typical S-band photoinjectors.

\section{THE RELATION BETWEEN PROJECTED EMITTANCE AND SLICE EMITTANCE}

The projected emittance can be regarded as composed of correlated emittance and slice emittance $[29,30]$. The correlated emittance describes the degree of coincidence of the phase space of the temporal slices, which can be caused by the phase angle mismatch and the beam size mismatch [31]. The slice emittance includes the thermal emittance and the emittance growth due to the nonlinearity of the transverse position and momentum. Correlation between transverse forces and longitudinal coordinate $z$ results in the evolution of the correlated emittance, and correspondingly correlation between transverse forces and radial coordinate $r$ results in the evolution of the slice emittance.

Our analysis is based on a VHF gun photoinjector, as depicted in Fig. 1. The photoinjector is a VHF gun test beamline designed for the diagnosis and optimization of the electron beam produced by a VHF gun. The VHF gun, which is manufacturing at Tsinghua University, is a singlecell copper rf cavity operating in $\mathrm{CW}$ mode at $216.67 \mathrm{MHz}$. Following the VHF gun, there are two solenoids for the emittance compensation and beam focusing. Between the two solenoids, there is a room-temperature $\mathrm{CW} 1.3 \mathrm{GHz}$ buncher cavity for the ballistic compression of the electron beam. After the solenoids and buncher, there are two $1.3 \mathrm{GHz}$ accelerating tubes (ACC) to accelerate the beam to $30 \mathrm{MeV}$.

A start-to-end beam dynamics simulation of the photoinjector is performed in Astra [32]. The number of macroparticles is 200000 . The bunch is divided into 40 slices when calculating the slice emittance, and each slice contains 5000 macroparticles. After beam optimizations in the genetic algorithm, the final projected emittance at the end of the injector is $\epsilon_{x}=0.173 \mathrm{mmmrad}$ and $\epsilon_{r}=0.261 \mathrm{~mm} \mathrm{mrad}$. The key parameters of the beamline achieving the best beam quality are shown in Table I.

The radial normalized emittance is employed in the following analysis considering that the photoinjector is axisymmetric. As derived in the Appendix, $\epsilon_{r}=2 \epsilon_{x}$ if thermal emittance is zero, and the thermal emittance $\epsilon_{\text {thermal, } \mathrm{r}}=\sqrt{2} \epsilon_{\text {thermal, } \mathrm{x}}$ at the cathode. The projected emittance can be written as

$$
\epsilon_{\text {projected,r }}=\beta \gamma \sqrt{\left\langle r^{2}\right\rangle\left\langle r^{\prime 2}\right\rangle-\left\langle r r^{\prime}\right\rangle^{2}}
$$


TABLE I. Key parameters of the VHF gun test beamline designed by Tsinghua University.

\begin{tabular}{lcc}
\hline \hline Parameters & Values & Units \\
\hline Charge & 100 & $\mathrm{pC}$ \\
Thermal emittance & 1 & $\mathrm{umrad} / \mathrm{mm}$ \\
Laser duration (flattop) & 48.5 & $\mathrm{ps}$ \\
Laser diameter (uniform) & 0.48 & $\mathrm{~mm}$ \\
Maximum gun field & 30 & $\mathrm{MV} / \mathrm{m}$ \\
Center of SOL1 & 0.303 & $\mathrm{~m}$ \\
Maximum SOL1 strength & 585.8 & $\mathrm{Gs}$ \\
Center of the buncher & 1 & $\mathrm{~m}$ \\
Bunch voltage & 336 & $\mathrm{kV}$ \\
Buncher phase & -45 & $\mathrm{deg}$ \\
Center of SOL2 & 2.14 & $\mathrm{~m}$ \\
Maximum SOL2 strength & 440.4 & $\mathrm{Gs}$ \\
Beginning of ACC & 2.85 & $\mathrm{~m}$ \\
\hline \hline
\end{tabular}

where $r$ and $r^{\prime}$ are, respectively, the radial position and divergence angle of a particle relative to the longitudinal axis $z$, and the average is performed over the whole bunch. $\beta$ and $\gamma$ are Lorentz factors.

The slice emittance $\epsilon_{\text {slice, r }}$ is in the same form as $\epsilon_{\text {projected,r }}$ but the average is performed over only one slice.

The evolution curve of the projected emittance and the emittances of several slices in the VHF gun photoinjector are shown in Fig. 2. It can be found that the emittance evolution curve of different slices is similar, but quite different from the projected emittance, which is because in the contributions of the projected emittance the correlated emittance occupies a large proportion, and the correlated emittance and the slice emittance evolute independently.

The correlated emittance can be expressed as

$$
\epsilon_{\text {correlated,r }}=\beta \gamma \sqrt{\left\langle\sigma_{r}^{2}\right\rangle\left\langle\sigma_{r}^{\prime 2}\right\rangle-\left\langle\sigma_{r} \sigma_{r}^{\prime}\right\rangle^{2}}
$$

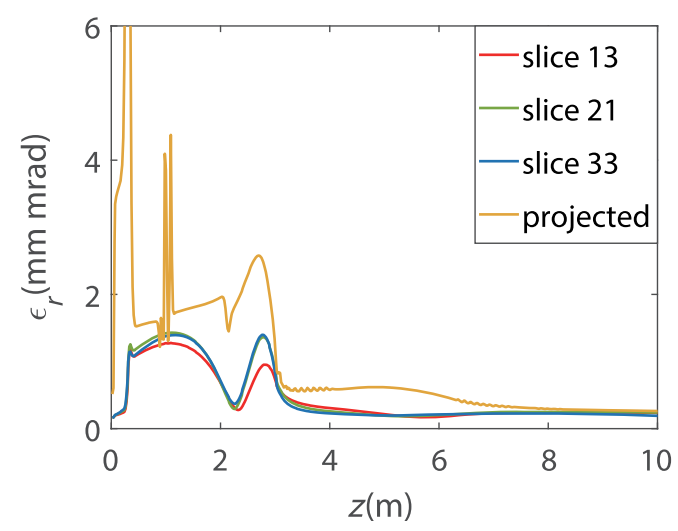

FIG. 2. The evolution of slice emittance and projected emittance in the VHF gun injector.

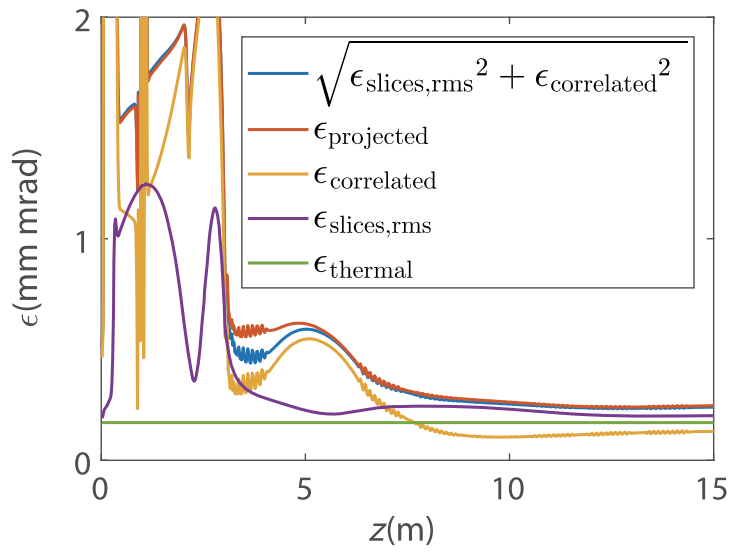

FIG. 3. The relation between slice emittance, correlated emittance, and projected emittance.

where $\sigma_{r}=\left\langle r^{2}\right\rangle$ is the radial rms beamsize of a slice and $\sigma_{r}^{\prime}=\frac{\left\langle r^{\prime}\right\rangle}{\sqrt{\left\langle r^{2}\right\rangle}}$ is the derivative to longitudinal axis of $\sigma_{r}$. The average is performed over all slices.

The evolution curves of the correlated emittance and the projected emittance are shown in Fig. 3 as the yellow and the red lines, respectively. Besides, the rms value of the emittances of all slices, which is expressed as

$$
\epsilon_{\text {slices }, \mathrm{rms}}=\sqrt{\frac{1}{n}\left(\epsilon_{\text {slice }, 1}^{2}+\epsilon_{\text {slice }, 2}^{2}+\cdots+\epsilon_{\text {slice }, \mathrm{n}}^{2}\right)},
$$

are shown in the purple line in Fig. 3. The slice emittance of a slice $i$ can be written as $\epsilon_{i}^{2}=\beta \gamma\left(\left\langle r^{2}\right\rangle_{i}\left\langle r^{\prime 2}\right\rangle_{i}-\right.$ $\left.\left\langle r r^{\prime}\right\rangle_{i}^{2}\right)=\beta \gamma\left(\sigma_{i}^{2}\left\langle r^{\prime 2}\right\rangle_{i}-\sigma_{i}^{2} \sigma_{i}^{\prime 2}\right)$. Take the average over all slices $\left\langle\epsilon_{i}^{2}\right\rangle=\beta \gamma\left(\left\langle\sigma_{i}^{2}\left\langle r^{\prime 2}\right\rangle_{i}\right\rangle-\left\langle\sigma_{i}^{2} \sigma_{i}^{\prime 2}\right\rangle\right)$. Then $\epsilon_{\text {projected }}^{2}=$ $\beta \gamma\left(\left\langle r^{2}\right\rangle\left\langle r^{\prime 2}\right\rangle-\left\langle r r^{\prime 2}\right\rangle\right)=\beta \gamma\left(\left\langle\sigma_{i}^{2}\right\rangle\left\langle r^{\prime 2}\right\rangle-\left\langle\sigma_{i} \sigma_{i}^{\prime}\right\rangle^{2}\right)=\left\langle\epsilon_{i}^{2}\right\rangle+$ $\beta \gamma\left(\left\langle\sigma_{i}^{2} \sigma_{i}^{\prime 2}\right\rangle-\left\langle\sigma_{i} \sigma_{i}^{\prime}\right\rangle^{2}+\left\langle\sigma_{i}^{2}\right\rangle\left\langle r^{\prime 2}\right\rangle-\left\langle\sigma_{i}^{2}\left\langle r^{\prime 2}\right\rangle_{i}\right\rangle\right)=\left\langle\epsilon_{i}^{2}\right\rangle+$ $\epsilon_{\text {correlated }}^{2}+\beta \gamma\left(\left\langle\sigma_{i}^{2}\right\rangle\left\langle r^{\prime 2}\right\rangle-\left\langle\sigma_{i}^{2}\left\langle r^{\prime 2}\right\rangle_{i}\right\rangle\right)$. When the square of the slice rms beamsize is irrelevant to the square of the slice rms divergence, we have

$$
\left\langle\sigma_{i}^{2}\right\rangle\left\langle r^{\prime 2}\right\rangle=\left\langle\sigma_{i}^{2}\left\langle r^{\prime 2}\right\rangle_{i}\right\rangle
$$

In this case, the projected emittance is composed of two independent parts, correlated emittance and slice emittance, i.e., $\epsilon_{\text {projected }} \cong \sqrt{\left\langle\epsilon_{i}^{2}\right\rangle+\epsilon_{\text {correlated }}^{2}}=\sqrt{\epsilon_{\text {slices,rms }}^{2}+\epsilon_{\text {correlated }^{2}}}$ (blue line in Fig. 3).

It can be found that the blue line is very close to the projected emittance (red line), indicating that Eq. (4) is approximately true in our simulation. At the end of the photoinjector the thermal emittance, the slice emittance excluding the thermal emittance and the correlated emittance are $0.17,0.128,0.151 \mathrm{~mm} \mathrm{mrad}$, respectively, revealing that the slice emittance (especially the thermal emittance) accounts for a large proportion of the final projected emittance. In the following section, an analysis of 
the slice emittance evolution in the photoinjector is demonstrated by studying the contributions of different radial forces to the beam momentum change.

\section{ANALYSIS ON THE EVOLUTION OF THE SLICE EMITTANCE}

\section{A. Photo-gun section}

The photo-gun is a key component of a injector, which boosts the beam velocity from almost rest to near the speed of light in a short distance. In this process, SC force, inversely proportional to the square of $\gamma$, has significant influence on beam quality. It is well known that uniform three-dimensional ellipsoidal distribution of electron beam can cancel the nonlinearity due to SC forces [33]. However, the three-dimensional ellipsoidal distribution is difficult to produce, and the longitudinal uniform distribution, as a compromise, is more commonly used in most of the photoinjectors. For a longitudinal uniform distribution with a transverse Gaussian-cut distribution, the charge density $\rho$ can be expressed

$\rho=\frac{Q \exp \left(-\frac{x^{2}+y^{2}}{2 \sigma_{x}^{2}}\right)}{2 \pi \sigma_{x}^{2} \operatorname{Erf}\left(R /\left(\sqrt{2} \sigma_{x}\right)\right)^{2} l}, x^{2}+y^{2}<R^{2},-\frac{l}{2}<z<\frac{l}{2}$,

where $Q$ is the bunch charge, $R$ is the beam radius, and $l$ is the longitudinal length. $\frac{R}{\sigma_{x}}=1$ means $1 \sigma$ Gaussian-cut. Small $\frac{R}{\sigma_{x}}$ like 0.1 means nearly uniform distribution. The transverse electric field $E_{x}$ can be written as

$$
\begin{aligned}
E_{x}(x, y, z)= & \frac{1}{4 \pi \varepsilon_{0}} \frac{\partial}{\partial x} \int \frac{\rho\left(x^{\prime}, y^{\prime}, z^{\prime}\right) d x^{\prime} d y^{\prime} d z^{\prime}}{\sqrt{\left(x-x^{\prime}\right)^{2}+\left(y-y^{\prime}\right)^{2}+\left(z-z^{\prime}\right)^{2}}} \\
& x^{\prime 2}+y^{\prime 2}<R^{2},-\frac{l}{2}<z^{\prime}<\frac{l}{2}
\end{aligned}
$$

Based on the ratio of transverse and longitudinal dimensions, the beam with longitudinal uniform distribution can be divided into three categories: pancake-like bunch $\left(\frac{l}{R} \ll 1\right)$, normal bunch $\left(\frac{l}{R}\right.$ is close to 1$)$, and cigarlike bunch $\left(\frac{l}{R} \gg 1\right)$. Typically pancake-like bunch is more commonly used in S- or shorter band rf guns. However, the VHF gun prefers cigar-like bunch because the wavelength of VHF band is much longer than the S-band, and the rf emittance in the VHF gun is negligible even for longer bunches.

Feng Zhou [25,34] has proven that transverse $1 \sigma$ Gaussian-cut distribution is better than transverse uniform distribution for a pancake-like bunch. In this section, we will demonstrate that the transverse uniform distribution is better than the transverse Gaussian-cut distribution for a cigar-like bunch. The transverse SC field $E_{x}$ [Eq. (6) with $y$, $z=0]$ as a function of $x / R$ is calculated for different beam
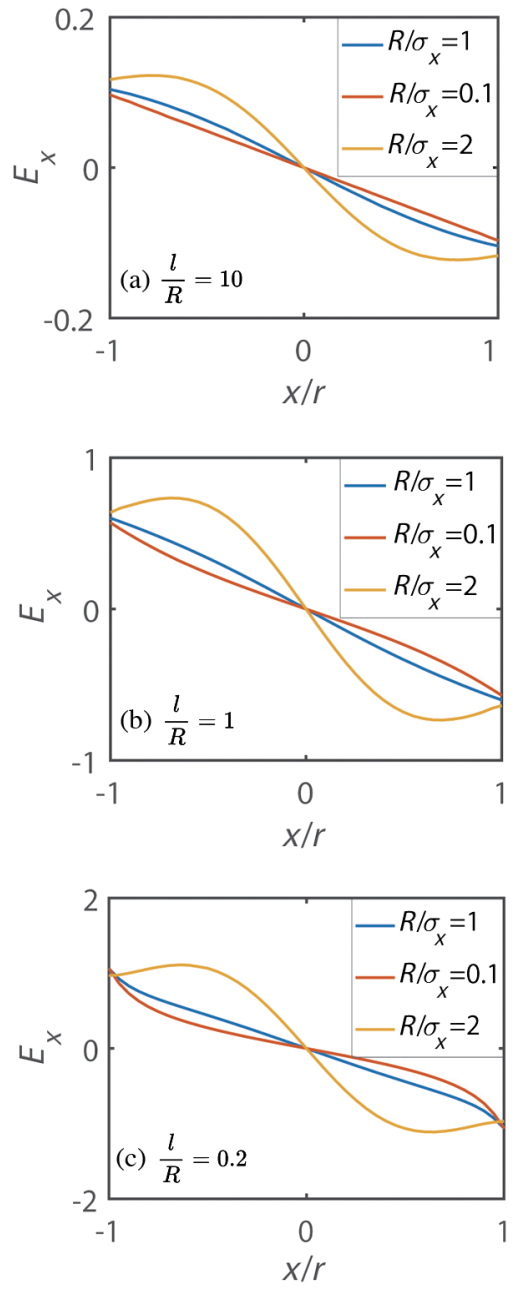

FIG. 4. Nonlinearity of transverse SC fields with different transverse Gaussian-cut distributions. $R / \sigma_{x}=0.1$ indicates an approximately uniform distribution, $R / \sigma_{x}=1$ or 2 indicates a Gaussian distribution. (a) cigar-like bunch, $\frac{l}{R}=10$. (b) normal bunch, $\frac{l}{R}=1$. (c) pancake-like bunch, $\frac{l}{R}=0.2$.

shapes, as shown in Fig. 4. Three typical dimension ratios of electron bunch are demonstrated: (a) cigar-like bunch $\left(\frac{l}{R}=10\right)$, (b) normal bunch $\left(\frac{l}{R}=1\right)$, and (c) pancake-like bunch $\left(\frac{l}{R}=0.2\right)$. In each dimension ratio, $E_{x}$ of three different Gaussian-cut distributions, i.e., $R / \sigma_{x}=0.1,1$, and 2, respectively, are illustrated in Fig. 4. It can be found that the transverse SC force $\left(E_{x}\right)$ with transverse $1 \sigma$ Gaussian-cut distribution is more linear than the uniform distribution for pancake-like bunch and normal bunch. However, $E_{x}$ with uniform distribution is more linear than the Gaussian-cut distribution for a cigar-like bunch, based on our calculations illustrated in Fig. 4(a). For the VHF gun photoinjector shown in Sec. II, a cigar-like bunch is produced on the cathode. A simple calculation indicates that $\frac{l}{R}=16$ when all particles just leave the cathode. Therefore, initial beam with transverse uniform distribution is better than Gaussian distribution in VHF gun 

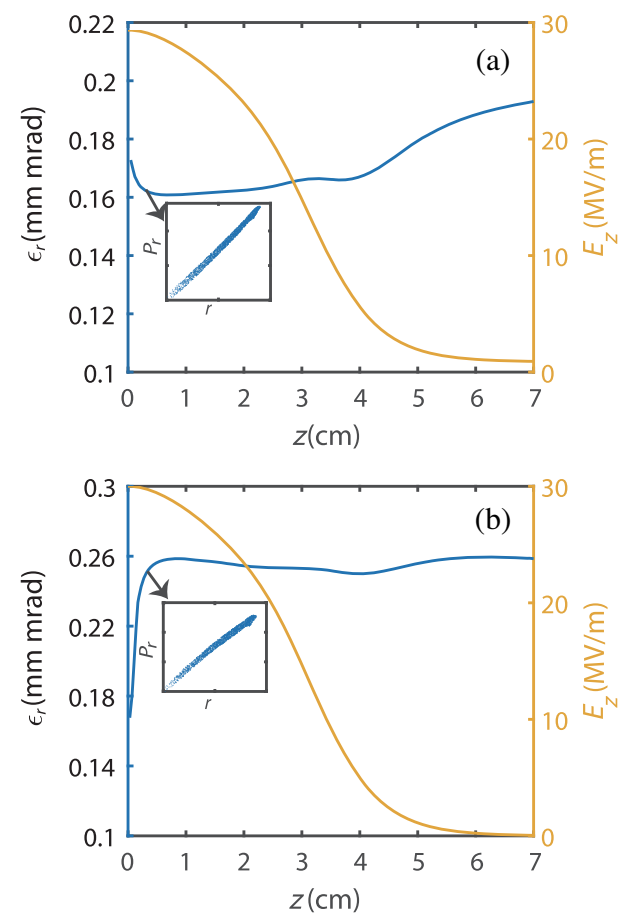

FIG. 5. Emittance evolution curves of the 21st slice in the gun with (a) initial transverse uniform distribution and (b) initial transverse $1 \sigma$ Gaussian-cut distribution. The yellow line indicates the $E_{z}$ field on the axis of the VHF gun. The inset displays the transverse phase space of the slice at position $3.1 \mathrm{~mm}$, where all particles in the bunch just leave the cathode.

photoinjector to suppress the emittance growth due to the nonlinear SC effects.

Furthermore, we provide the emittance evolution curves in the gun with two different initial transverse distributions, one is uniform distribution and the other is $1 \sigma$ Gaussian-cut distribution. The center (21st) slice of the bunch is employed as an example, as shown in Fig. 5. The yellow line indicates the $E_{z}$ field on the axis of the VHF gun. In the inset, we plot the transverse phase space of the slice at position $3.1 \mathrm{~mm}$, where all particles in the bunch just leave the cathode. It can be found that the phase space for the uniform distribution is more linear than the $1 \sigma$ Gaussiancut distribution. Moreover, the slice emittance increases rapidly from the cathode to $3.1 \mathrm{~mm}$ for the $1 \sigma$ Gaussian-cut distribution, but remains basically unchanged for the uniform distribution. The evolutions of the phase space and slice emittance reveal that the uniform distribution is better than the Gaussian-cut distribution, which is consistent with the conclusion based on the SC field analysis.

The integral momentum of each particle in the 21 st slice from the rf force can be calculated by tracing the electromagnetic field in the gun along the particle trajectory exported from ASTRA simulation. The calculated integral momentum as a function of the radial position of each particle at the gun exit is shown as the blue dots in Fig. 6 . The integral momentum contribution of the rf force is

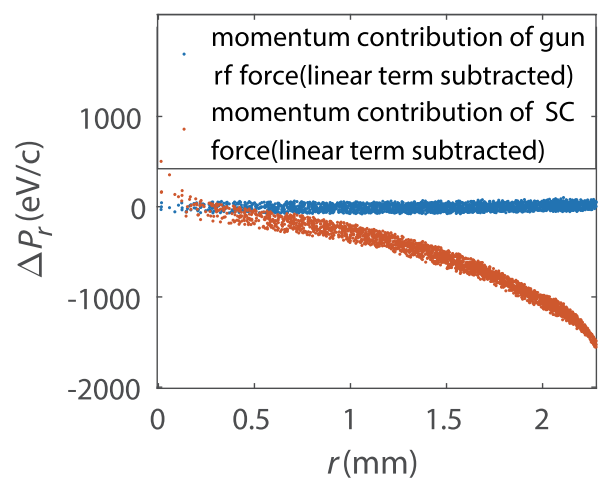

FIG. 6. The integral momentum of the particles as a function of the radial position at the gun exit. The integral momentum is calculated from the position where all particles are just emitted to the gun exit based on two independent forces: (1) gun rf force (blue) and (2) SC force (red). The linear terms of the integral momentum are subtracted.

computed from the position where all particles are just emitted to the gun exit. The linear term is subtracted. It can be found that the high-order integral momentum is very small, indicating that the emittance growth due to the $\mathrm{rf}$ force is negligible. Moreover, the integral momentum contribution of the SC force can be calculated by subtracting the initial momentum on the cathode and the integral momentum contribution of the rf force from the final momentum at the gun exit. The integral momentum contribution of the SC force as a function of the radial position of each particle at the gun exit is shown as the red dots in Fig. 6. The linear term is also subtracted. The curvature of the high-order integral momentum indicates an emittance growth due to the SC force.

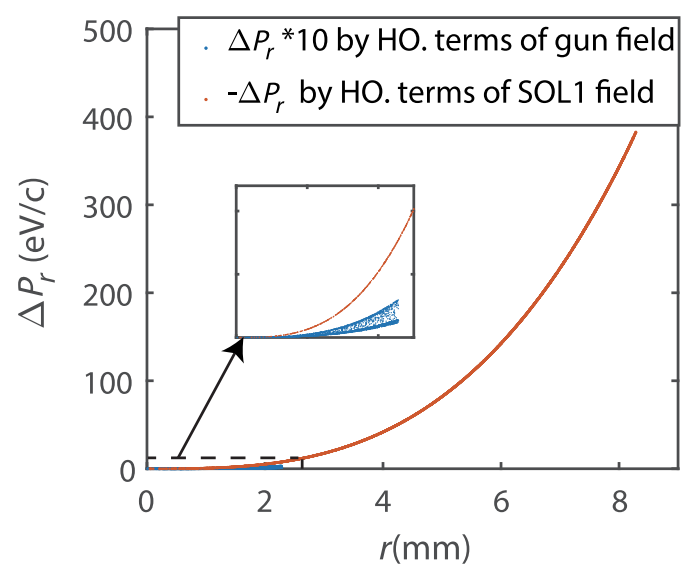

FIG. 7. The blue dots: the integral momentum contribution of the high-order terms of the rf field as a function of the radial position of each particle at the gun exit. The red dots: the integral momentum contribution of the high-order terms of the SOL1 field as a function of the particles' radial position at the end of the SOL1 field. Note that the momentum contribution of the rf field is magnified by 10 times in order to compare with the momentum contribution of the SOL1 field. 
The integral momentum contributions of the high-order terms of the rf field and SOL1 field are calculated by ignoring the linear part of the above fields, as shown in Fig. 7. It can be found that the nonlinearity of the slice phase space contributed by the gun field is very small compared with the high-order terms of the solenoid field. A start-to-end simulation illustrates that the projected emittance increases only 5.8\% when the high-order terms of all rf fields (gun, buncher, and ACCs), instead of the field firstorder approximation, are considered in the simulation. The high-order terms of the solenoid field are an important source of the slice emittance growth, which will be demonstrated in Sec. III B.

\section{B. Solenoid section}

Solenoid is a key component in a photoinjector for the beam focusing and the compensation of the correlated emittance. Due to the high-order terms of the solenoid field, nonlinearity will be introduced into the slice phase space, resulting in an increase of the slice emittance, and this phenomenon is called spherical aberration. Longitudinal magnetic field of an axisymmetric solenoid can be described as $B_{z}(r)=B_{z, 0}-\frac{r^{2}}{4} B_{z, 0}^{\prime \prime}+\cdots$, where $B_{z, 0}$ is the longitudinal on axis magnetic field and higher order terms above third order are omitted. According to Busch's theorem [35] $\gamma m r^{2} \dot{\theta}=-\pi r^{2} q B_{z, 0} / 2 \pi$ and equation of motion $\gamma m \ddot{r}=\gamma m r \dot{\theta}^{2}+q r \dot{\theta} B_{z, 0}$, we have

$$
\ddot{r}=-\frac{q^{2} B_{z, 0}^{2}}{4 \gamma^{2} m^{2}}\left[r-\frac{B_{z, 0}^{\prime \prime}}{2 B_{z, 0}} r^{3}\right],
$$

with higher order terms neglected. Under the thin-lens approximation ( $r$ remains unchanged in the solenoid), the change of velocity and momentum in $r$ direction can be expressed as [36]

$$
\begin{aligned}
& \Delta r^{\prime}=-\frac{1}{f_{0}}\left(r+C_{1} r^{3}\right) \quad \text { and } \\
& \Delta P_{r}=-\frac{\beta \gamma m c}{f_{0}}\left(r+C_{1} r^{3}\right),
\end{aligned}
$$

where $\frac{1}{f_{0}}=\left(\frac{q}{2 \beta \gamma m c}\right)^{2} \int_{-\infty}^{+\infty} B_{z, 0}^{2} d z$ and $r^{\prime}=d r / d z . \quad C_{1}=$ $\frac{1}{2} \int_{-\infty}^{+\infty} B_{z, 0}^{\prime} d z$ is the spherical aberration coefficient, which is only determined by the geometry of the solenoid.

Hence, the emittance growth can be written as

$$
\epsilon_{\Delta}=\sqrt{\left\langle r^{2}\right\rangle\left\langle\Delta P_{r}^{2}\right\rangle-\left\langle r \Delta P_{r}\right\rangle^{2}}=\frac{\sqrt{2} \beta \gamma m c C_{1} \sigma_{r}^{4}}{4 f_{0}},
$$

by assuming that the beam is transversely uniform with rms spot size of $\sigma_{r}$. Based on Eq. (10), it can be found that the

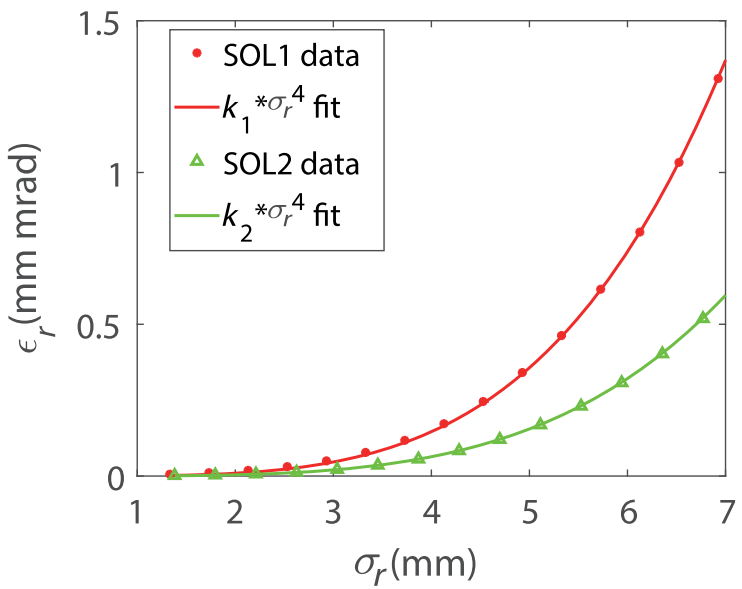

FIG. 8. Emittance growth as a function of the rms beam size at the solenoid center for SOL1 (red) and SOL2 (green). The points are the simulated data in ASTRA. The lines are the fitting curves of $\epsilon_{r}=k * \sigma_{r}^{4}$.

emittance growth due to the solenoid spherical aberration is proportional to the fourth power of the radius.

The emittance growth due to the solenoid spherical aberration is simulated in Astra. Two solenoids in the VHF gun photoinjector (SOL1 and SOL2 in Sec. II) are involved in the simulation. First, the emittance growth in SOL1 and SOL2 is evaluated independently with a simplified model. We assume that the initial beam before the solenoid has zero emittance, uniform transverse distribution, and perfectly parallel rays. The particles in the beam have identical momentum, which is the same as the beam momentum in SOL1 or SOL2 in the VHF gun photoinjector in Sec. II. The solenoid parameters used here are also the same as the optimized parameters listed in Table I. The initial beam spot size before the solenoid changes and the emittance growth after the beam passes through the solenoid are evaluated. The emittance growth varying with the rms beam size at the center of SOL1 and SOL2 is shown in Fig. 8. The simulated points are fitted using the function of $\epsilon_{r}=k * \sigma_{r}^{4}$, and the fitting curve is in good agreement with the data points, which is consistent with the theoretical description in Eq. (10).

Furthermore, the evolutions of the slice emittance and the rms beam size in SOL1 and SOL2 of the VHF gun photoinjector are illustrated using the 21st slice as an example, as shown in Fig. 9. The slice emittance evolutions with and without the solenoid are shown as the blue solid and purple dotted lines, respectively. The rms beam size in the center of SOL1 is $6 \mathrm{~mm}$ and there is a steep slice emittance growth in SOL1 due to the solenoid spherical aberration. Moreover, the rms beam size in the center of SOL2 is $3.1 \mathrm{~mm}$ and the emittance growth in SOL2 due to the spherical aberration is negligible. Therefore, the emittance growth due to the spherical aberration mainly occurs in SOL1. 

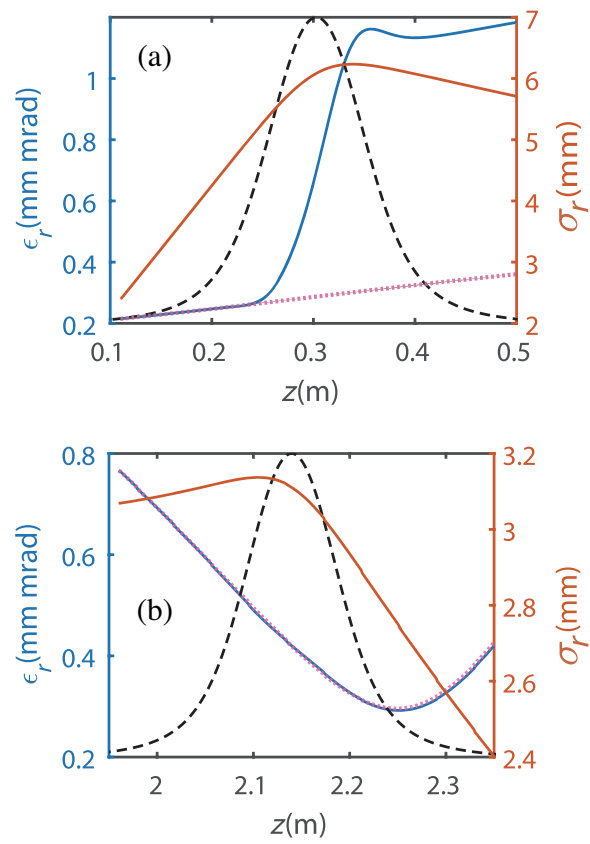

FIG. 9. The evolution curves of the 21st slice in (a) SOL1 and (b) SOL2. The black dashed lines are the on axis solenoid field. The blue and red solid lines indicate the evolution curves of the emittance and the rms beam size in the solenoids, respectively. As a comparison, the purple dotted lines indicate the emittance evolution curves without the solenoids.

Figure 10 demonstrates the integral momentum contribution of the magnetic field of SOL1 and SOL2. The linear term of integral momentum is subtracted. It can be found that the nonlinear momentum contribution of SOL1 is much larger than SOL2, which is consistent with the emittance evolutions in Fig. 9. The integral momentum of SOL1 with the linear term subtracted is fitted using the function of $\Delta P_{r}=b r^{3}$, and the fitting curve is in good agreement with the data points, which is consistent with the theoretical description in Eq. (9).

Based on Eq. (10), the emittance growth is proportional to the solenoid spherical aberration coefficient $C_{1}$, and $C_{1}$ is only determined by the solenoid field profile. Here, we demonstrate that the emittance growth due to spherical aberration can be suppressed by optimizing the solenoid field profile. The full width at half maximum (FWHM) of the SOL1 on axis $B_{z}$ field is scanned in ASTRA simulation. The strengths of the two solenoids and the position of SOL2 are optimized to obtain the optimal projected emittance at the end of the photoinjector. The emittance growth due to $B_{z}$ on the cathode and the optimal projected emittance are shown in Fig. 11. It can be found that the emittance growth due to $B_{z}$ on the cathode increases when the field width increases. Even so, the optimal projected emittance decreases with increasing field width, indicating that the spherical aberration decreases as the field width increases.
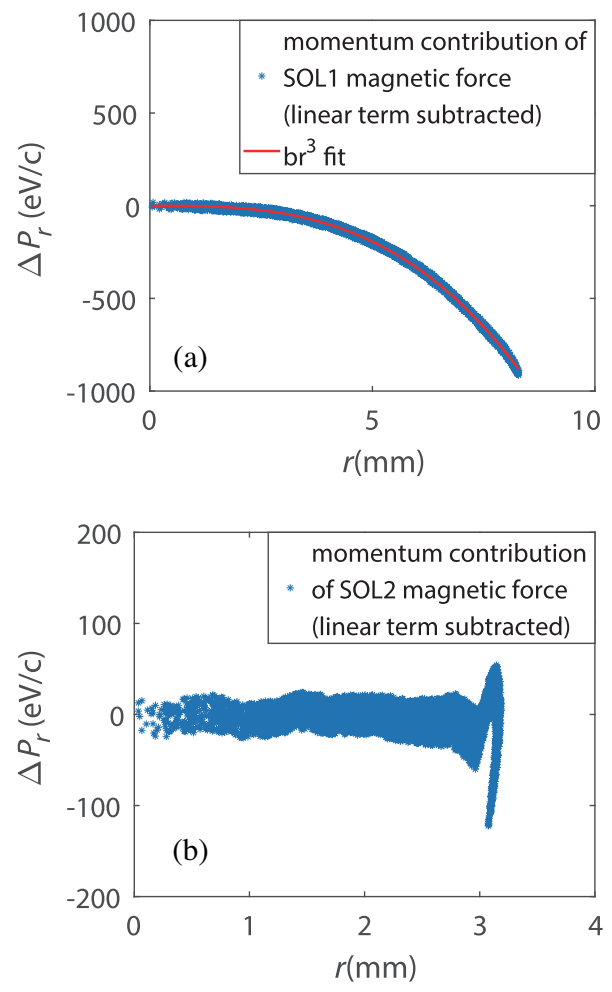

FIG. 10. The integral momentum contribution of the magnetic field of (a) SOL1 and (b) SOL2 as a function of the radial position of each particle at the end of the solenoid field. The linear term of the integral momentum is subtracted. The red line is a fitting curve of $\Delta P_{r}=b r^{3}$.

\section{Slice emittance self-reducing effects in drift section}

In Sec. III A, we have illustrated that the emittance growth near the cathode is mainly due to the nonlinear SC forces. In this section, however, we will demonstrate that the nonlinear SC forces do not necessarily lead to a growth of the slice emittance for an electron beam moving in a drift

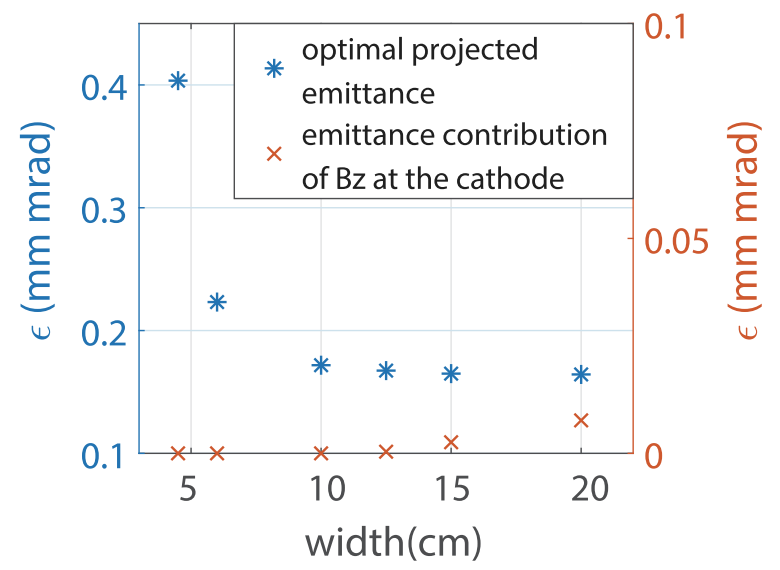

FIG. 11. The emittance growth due to $B_{z}$ on the cathode and the optimal projected emittance as a function of the FWHM of the SOL1 field. 


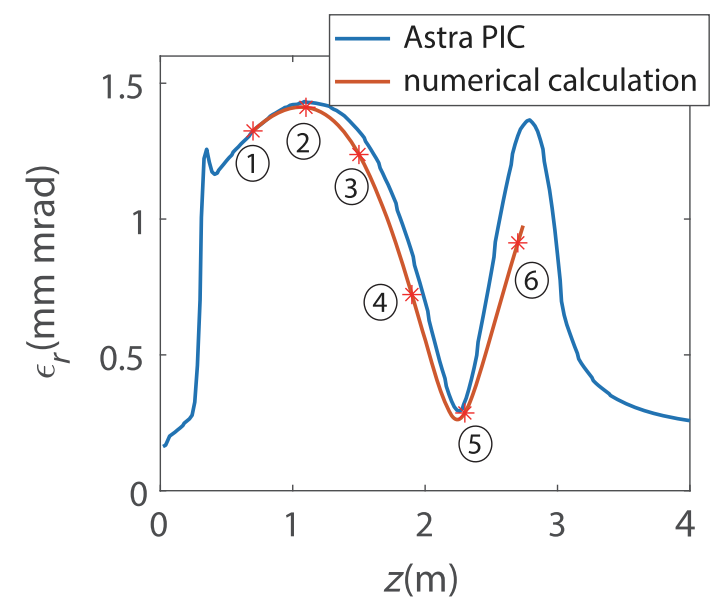

FIG. 12. The blue line indicates the emittance evolution of the 21st slice simulated in Astra. The red line is the slice emittance evolution calculated based on Eq. (11) using the particle information at $0.7 \mathrm{~m}$ exported from Astra as initial conditions.

section. Under certain conditions, the slice emittance will decrease under the effects of the nonlinear SC force, and this phenomenon is called self-linearization of the phase space or self-reducing of the slice emittance. This effect has been mentioned in some works $[31,37,38]$ and explained in Ref. [38] based on simple physical model. In our work, we study the self-reducing effect from the perspective of the transverse distribution evolution of the electron beam based on our VHF gun photoinjector.

The slice emittance evolution from the cathode to the position of $4 \mathrm{~m}$ (in the first ACC) is shown in Fig. 12 using the 21st slice as an example. We choose a section of our VHF gun photoinjector to study the self-reducing effect of the nonlinear SC forces. This section is from the end of the SOL1 field $(0.7 \mathrm{~m})$ to the entrance of the first ACC $(2.85 \mathrm{~m})$. In this section, there is a SOL2 and a buncher besides the drift. However, we already know from Sec. III B that the emittance growth due to the SOL2 is negligible. In addition, we will also explain in Sec. III D that the buncher has a very small effect on the slice emittance. Therefore, this section can be viewed as a simple drift section only under the effect of the SC forces.

The transverse SC force inside the slice can be described as

$$
r^{\prime \prime}=\frac{e \frac{n(r)}{n_{0}} \frac{q}{l}}{2 \pi \varepsilon_{0} r \beta^{2} \gamma^{3} m c^{2}},
$$

where $\frac{q}{l}$ is the charge per unit length, $e$ and $m$ are the electron charge and rest mass, respectively. $\frac{n(r)}{n_{0}}$ is the proportion of electrons within radius $r$.

The information of the particles' position and momentum at $0.7 \mathrm{~m}$ is exported from ASTRA simulation. Using this information as the initial condition, we can calculate
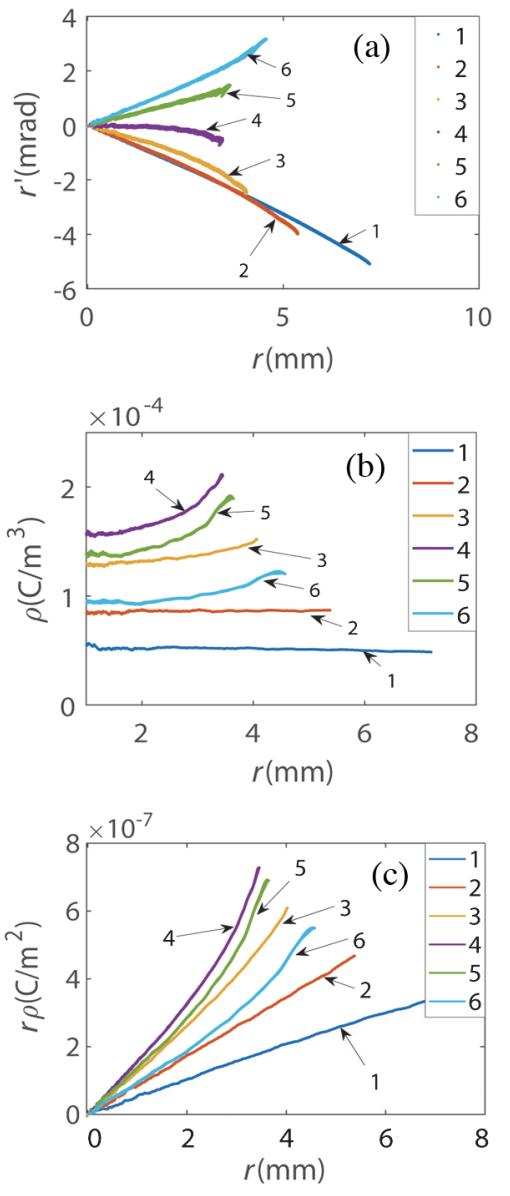

FIG. 13. (a): The $r-r^{\prime}$ phase space, (b): The average charge density per unit volume within the radius $r$, (c): $r \rho$ as a function of r. The number 1 to 6 in the figure correspond to the positions marked in Fig. 12.

the slice emittance evolution with only the SC forces based on Eq. (11), and the calculated emittance evolution is shown as the red line in Fig. 12. It can be found that the numerical calculation of the slice emittance evolution with only the SC forces is very close to the real evolution in ASTRA simulation, proving that the SC force dominates the emittance evolution and the effects of the SOL2 and the buncher are negligible. Moreover, the slice emittance is oscillating under the effect of the nonlinear SC force. We choose six positions in the photoinjector (marked in Fig. 12) to explain this oscillation phenomenon. The evolution of the slice phase space of the six positions is displayed in Fig. 13(a). The evolution of the transverse charge distribution is also displayed in Fig. 13(b). Here, the transverse charge distribution is described as the average charge density per unit volume within the radius $r$ :

$$
\rho=\frac{\frac{n(r)}{n_{0}} q}{\pi r^{2} l}
$$


It should be noted that

$$
r^{\prime \prime} \propto r \rho,
$$

thus $r \rho$ can be used to describe the change of the slice phase space in a short distance and also the strength of SC force. The evolution of $r \rho$ of the six positions is displayed in Fig. 13(c).

Point 1 is at the end of the SOL1 field $(0.7 \mathrm{~m})$. The charge distribution of point 1 is very uniform [see Fig. 13 (b)]. The slice phase space of point 1 indicates that the electron beam is being focused, and the phase space has a curvature, which is mainly caused by the spherical aberration of the SOL1. The curvature results in the electrons on the outer side moving to the inner side. Therefore, the evolutions of the charge distribution from point 1 to 5 exhibit that the charge density on the outer side becomes higher than the inner side, as shown in Fig. 13(b). The increasing curvature of the charge density from point 1 to 5 corresponds to an increasing curvature of $r \rho$, as shown in Fig. 13(c). From point 1 to $5 r \rho$ on the outer side is larger than the inner side, i.e., $\Delta r^{\prime}$ on the outer side is larger than the inner side based on Eq. (13). Therefore, the slice phase space becomes more and more straight, as shown in Fig. 13 (a). In summary, the curvature of the slice phase space causes the unevenness of the transverse charge distribution, and the nonlinear SC force due to the unevenness of the charge distribution in turn counteracts the curvature of the phase space. In this process, the nonlinear SC forces can actually compensate for the nonlinear transverse positionmomentum correlations and hence recover the emittance.

The phase space of point 5 becomes very straight and the slice emittance has reached the minimum point. However, the charge density $\rho$ remains uneven and the charge density on the outer side is higher than the inner side. This causes nonlinear SC forces as the curvature of $r \rho$ of point 5 in Fig. 13(c). Therefore, we can see that the phase space of point 6 becomes curved again, and then the slice emittance increases accordingly. After point 6 , the evolution of the slice emittance undergoes a second compensation. Due to the focusing of SOL2, the beam spot size during the second compensation is smaller, and the SC force is stronger, thus the slice emittance decreases faster than the first compensation. After the second compensation, the SC force decreases rapidly with the acceleration of the ACC. The phase space is frozen and the slice emittance is maintained near the second minimum point.

\section{Buncher and linac boosters}

The role of the buncher is to compress the beam length in the VHF gun photoinjector. Similar to the gun section in Sec. III A, the high-order integral momentum contribution of the buncher RF force is shown in Fig. 14(a), which is very small, indicating that the slice emittance growth due to the buncher RF force is negligible. The slice emittance
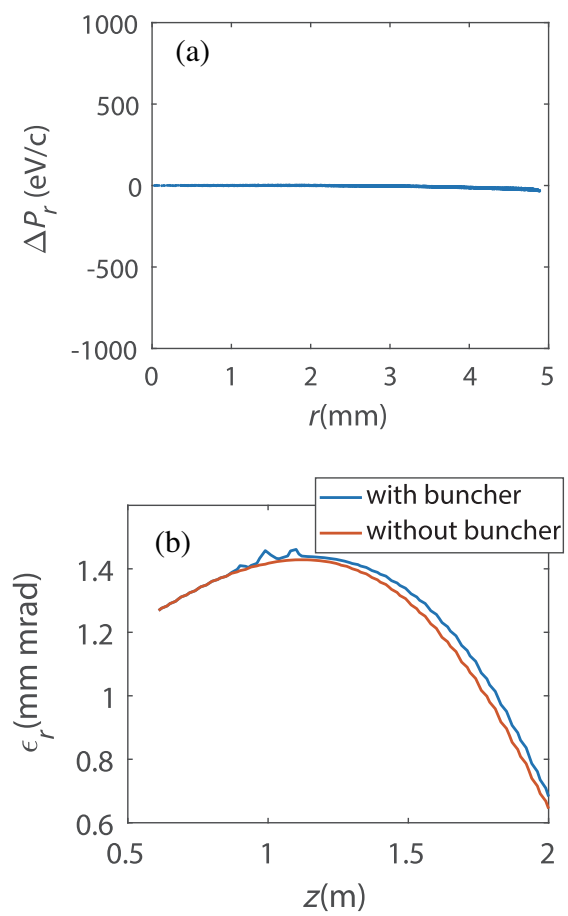

FIG. 14. (a) The high-order integral momentum contributions of the buncher rf force. (b) Slice emittance evolution with and without the buncher.

evolutions with and without the buncher are shown in Fig. 14(b). Compared with the case without the buncher, the slice emittance is slightly increased in the case with the buncher. This emittance growth is partly due to the nonlinear rf force of the buncher, and partly due to the larger $\mathrm{SC}$ force because of the beam longitudinal compression in the buncher.

The slice emittance evolutions with and without the linac boosters are shown in Fig. 15. The linac booster plays a role in freezing the emittance by increasing the Lorentz gamma factor of the electron beam. The final frozen best slice emittance is very close to the second minimum emittance without the linac boosters.

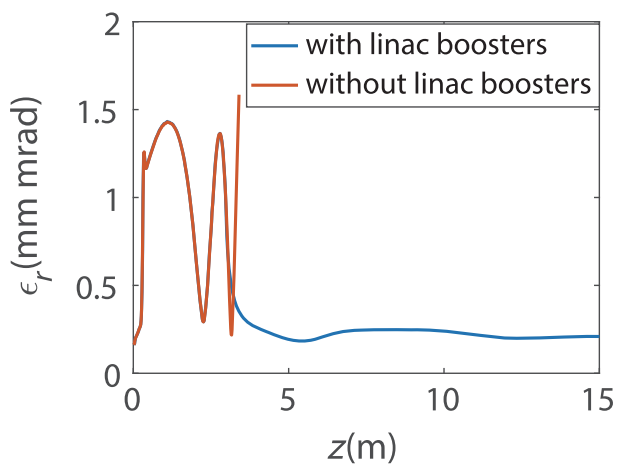

FIG. 15. The slice emittance evolutions with and without the linac boosters. 


\section{DISCUSSION}

We have provided a detailed analysis of the slice emittance evolution in a VHF gun photoinjector. Note that the analysis is based on an axisymmetric photoinjector beamline and nonideal factors are not taken into account. In fact, nonuniform emission due to cathode roughness [39] or transverse coupling due to skewed magnetic and radiofrequency quadrupole fields [40,41] will also result in the increase in slice emittance while it's beyond the scope of this paper. In this section, we will briefly demonstrate that similar analysis can be performed in other types of photoinjectors. Taking an S-band gun photoinjector as an example, the evolutions of the slice emittance and the projected emittance are shown in Fig. 16. The drive laser has a temporal plateau distribution with 5 ps FWHM and spatial uniform distribution with $0.6 \mathrm{~mm}$ radius. The bunch charge is $250 \mathrm{pC}$. The ratio of the bunch longitudinal length to the radius $\frac{l}{R}$ is 0.55 when all particles just leave the cathode. Therefore, the bunch is pancake-like and there is a strong nonlinear SC force when the bunch is just emitted from the cathode. From Fig. 16, we can see that at the vicinity of the cathode the slice emittance increases rapidly from the thermal emittance of 0.385 to $0.861 \mathrm{~mm}$ mrad in a short distance due to the nonlinear SC force. The center of the solenoid near the gun is at about $0.21 \mathrm{~m}$ from the cathode. Different from the VHF gun photoinjector, the S-band gun boosts the beam to a higher energy and the beam size in the solenoid is also smaller, thus the emittance growth due to the solenoid spherical aberration is small. ASTRA simulation shows that the emittance growth $\epsilon_{r}$ due to the solenoid is only $0.07 \mathrm{~mm}$ mrad. Similar to the VHF gun photoinjector, in the drift section downstream of the S-band gun the slice emittance reduces to the thermal emittance due to the selflinearizing effect of the nonlinear SC force, then the emittance oscillates until the emittance is frozen by the linacs. The frozen emittance is close to the second compensation point.

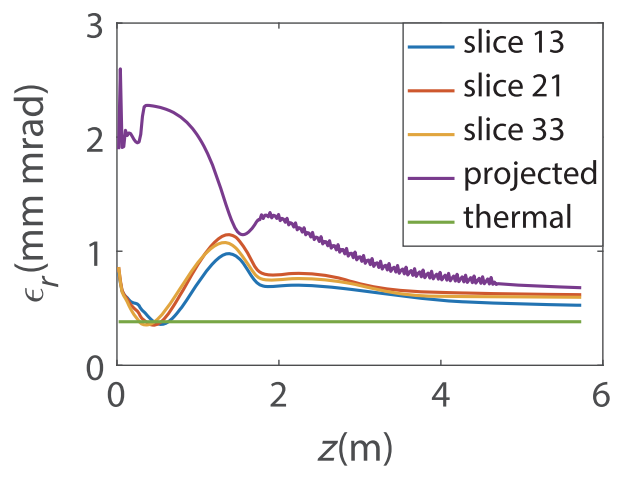

FIG. 16. The evolution of the slice emittance and the projected emittance in a typical S-band gun photoinjector. The green line indicates the thermal emittance.

\section{CONCLUSION}

The evolution of the slice transverse emittance in a VHF gun photoinjector has been systematically studied in this paper. First, the contributions of the slice emittance and the correlated emittance in the projected emittance are investigated individually based on theoretical analysis and beam dynamics simulations. We found that the slice emittance accounts for a large proportion of the final projected emittance. Second, the slice emittance evolution in different sections is studied one by one. The nonlinear SC force will lead to emittance growth near the cathode in the gun section. Based on SC force calculations, we found that the $1 \sigma$ Gaussian-cut distribution is better than the uniform distribution for a pacake-like or normal bunch. However, the uniform distribution is better than the Gaussian-cut distribution for a cigar-like bunch. Therefore, uniform distribution should be used in VHF gun photoinjectors because cigar-like bunch is usually adopted in this kind of photoinjector. The solenoid spherical aberration will result in significant slice emittance growth, especially in the solenoid close to the gun, because the beam size in this solenoid is large. By optimizing the solenoid field profile and reducing the solenoid spherical aberration, the optimal projected emittance can be reduced in the photoinjector. In the drift section, we found that the emittance oscillates under the effects of the nonlinear SC force. The nonlinear $\mathrm{SC}$ forces can actually compensate for the emittance growth due to the solenoid spherical aberration and recover the emittance. The slice emittance compensation was explained by analyzing the evolution of the phase space and the charge transverse distribution. The buncher has no significant effect on the slice emittance and the linac booster plays a role in freezing the slice emittance. We also demonstrate that the methodology and results reported here are also useful for other types of modern photoinjectors.

\section{APPENDIX: DERIVATION OF THE RELATION BETWEEN RADIAL EMITTANCE AND HORIZONTAL EMITTANCE}

If the thermal emittance is zero, i.e., the initial transverse momentum is zero, the particle momentum is always collinear with $r$ because the entire beam line is axisymmetric and no rotary motion is considered. $r^{\prime}$, which is the projection of particle momentum in $r$ direction, equals $\sqrt{x^{\prime 2}+y^{\prime 2}}$. Then

$$
\begin{aligned}
\epsilon_{r} & =\beta \gamma \sqrt{\left\langle r^{2}\right\rangle\left\langle r^{\prime 2}\right\rangle-\left\langle r r^{\prime}\right\rangle^{2}} \\
& =\beta \gamma \sqrt{\left\langle x^{2}+y^{2}\right\rangle\left\langle x^{\prime 2}+y^{\prime 2}\right\rangle-\left\langle\sqrt{\left(x^{2}+y^{2}\right)\left(x^{\prime 2}+y^{\prime 2}\right)}\right\rangle^{2}} .
\end{aligned}
$$


Because $x$ and $y$ are equivalent, we have $\left\langle x^{2}+y^{2}\right\rangle=$ $2\left\langle x^{2}\right\rangle$ and $\left\langle x^{\prime 2}+y^{\prime 2}\right\rangle=2\left\langle x^{\prime 2}\right\rangle$. Because the particle momentum is always collinear with $r$, we have $\frac{y}{x}=\frac{y^{\prime}}{x^{\prime}}=k$, where $k$ varies with different particles. Then $\left\langle\sqrt{\left(x^{2}+y^{2}\right)\left(x^{\prime 2}+y^{\prime 2}\right)}\right\rangle=\left\langle\left(1+k^{2}\right) x x^{\prime}\right\rangle=\left\langle x x^{\prime}+y y^{\prime}\right\rangle=2\left\langle x x^{\prime}\right\rangle$. We get $\epsilon_{r}=2 \epsilon_{x}$.

For the thermal emittance, $\epsilon_{x}=\beta \gamma \sqrt{\left\langle x^{2}\right\rangle\left\langle x^{\prime 2}\right\rangle}$ and $\epsilon_{r}=\beta \gamma \sqrt{\left\langle r^{2}\right\rangle\left\langle r^{\prime 2}\right\rangle}$. Here the particle momentum is not collinear with $r$ and $r^{\prime}=\frac{x x^{\prime}+y y^{\prime}}{\sqrt{x^{2}+y^{2}}}$. It is easy to get that $\left\langle r^{2}\right\rangle=2\left\langle x^{2}\right\rangle$. Because $x, x^{\prime}, y$, and $y^{\prime}$ are irrelevant to each other, $\left\langle r^{\prime 2}\right\rangle=\left\langle\frac{\left(x x^{\prime}+y y^{\prime}\right)^{2}}{x^{2}+y^{2}}\right\rangle=\left\langle\frac{x^{2} x^{\prime 2}+y^{2} y^{\prime 2}}{x^{2}+y^{2}}\right\rangle=\left\langle\frac{\left(x^{2}+y^{2}\right) x^{\prime 2}}{x^{2}+y^{2}}\right\rangle=\left\langle x^{\prime 2}\right\rangle$, so $\epsilon_{\text {thermal }, \mathrm{r}}=\sqrt{2} \epsilon_{\text {thermal, } \mathrm{x}}$.

[1] B. W. J. McNeil and N. R. Thompson, X-ray free-electron lasers, Nat. Photonics 4, 814 (2010).

[2] P. Emma et al., First lasing and operation of an ångstromwavelength free-electron laser, Nat. Photonics 4, 641 (2010).

[3] T. Ishikawa et al., A compact X-ray free-electron laser emitting in the sub-ångström region, Nat. Photonics 6, 540 (2012).

[4] H.-S. Kang et al., Hard X-ray free-electron laser with femtosecond-scale timing jitter, Nat. Photonics 11, 708 (2017).

[5] H. Weise et al., Commissioning and first lasing of the european XFEL, in Proceedings of 38th International Free Electron Laser Conference FEL'17 Santa Fe, NM, USA (2017).

[6] C. J. Milne et al., Swiss FEL: The Swiss X-ray Free Electron Laser, Appl. Sci. 7, 720 (2017).

[7] F. V. Hartemann, W. J. Brown, D. J. Gibson, S. G. Anderson, A. M. Tremaine, P. T. Springer, A. J. Wootton, E. P. Hartouni, and C. P. J. Barty, High-energy scaling of Compton scattering light sources, Phys. Rev. ST Accel. Beams 8, 100702 (2005).

[8] K. Deitrick, G. Krafft, B. Terzić, and J. Delayen, Highbrilliance, high-flux compact inverse Compton light source, Phys. Rev. Accel. Beams 21, 080703 (2018).

[9] S. M. Gruner, D. Bilderback, I. Bazarov, K. Finkelstein, G. Krafft, L. Merminga, H. Padamsee, Q. Shen, C. Sinclair, and M. Tigner, Energy recovery linacs as synchrotron radiation sources, Rev. Sci. Instrum. 73, 1402 (2002).

[10] C. Gulliford et al., Demonstration of low emittance in the cornell energy recovery linac injector prototype, Phys. Rev. ST Accel. Beams 16, 073401 (2013).

[11] A. H. Zewail, 4D ultrafast electron diffraction, crystallography, and microscopy, Annu. Rev. Phys. Chem. 57, 65 (2006).

[12] S. P. Weathersby et al., Mega-electron-volt ultrafast electron diffraction at SLAC National Accelerator Laboratory, Rev. Sci. Instrum. 86, 073702 (2015).

[13] A. H. Zewail, Four-Dimensional Electron Microscopy, Science 328, 187 (2010).
[14] R. K. Li and P. Musumeci, Single-shot MeV Transmission Electron Microscopy with Picosecond Temporal Resolution, Phys. Rev. Applied 2, 024003 (2014).

[15] D. Xiang, F. Fu, J. Zhang, X. Huang, L. Wang, X. Wang, and W. Wan, Accelerator-based single-shot ultrafast transmission electron microscope with picosecond temporal resolution and nanometer spatial resolution, Nucl. Instrum. Methods Phys. Res., Sect. A 759, 74 (2014).

[16] C. Gulliford, A. Bartnik, I. Bazarov, B. Dunham, and L. Cultrera, Demonstration of cathode emittance dominated high bunch charge beams in a dc gun-based photoinjector, Appl. Phys. Lett. 106, 094101 (2015).

[17] C. Hernandez-Garcia, T. Siggins, S. Benson, D. Bullard, H. Dylla, K. Jordan, C. Murray, G. Neil, M. Shinn, and R. Walker, A high average current dc gaas photocathode gun for ERLs and FELs, in Proceedings of the 2005 Particle Accelerator Conference, Knoxville, Tennessee (IEEE, 2005), pp. 3117-3119.

[18] D. Janssen et al., First operation of a superconducting rfgun, Nucl. Instrum. Methods Phys. Res., Sect. A 507, 314 (2003).

[19] R. Wells, W. Ghiorso, J. Staples, T. Huang, F. Sannibale, and T. Kramasz, Mechanical design and fabrication of the vhf-gun, the berkeley normal-conducting continuous-wave high-brightness electron source, Rev. Sci. Instrum. 87, 023302 (2016).

[20] Z. Wang, Q. Gu, M. Zhao, and G. Wang, Injector physics design at shine, in Proceedings of 10th International Particle Accelerator Conference (IPAC'19), Melbourne, Australia, 2019 (JACOW, Geneva, Switzerland, 2019), pp. 1801-1803.

[21] C. Papadopoulos, P. Emma, D. Filippetto, T. Raubenheimer, F. Sannibale, J. Schmerge, L. Wang, and F. Zhou, $\mathrm{Rf}$ injector beam dynamics optimization for LCLS-ii, in Proceedings of 5th International Particle Accelerator Conference (IPAC'14), Dresden, Germany, 2014 (JACOW, Geneva, Switzerland, 2014), pp. 1974-1976.

[22] R. Alley et al., The design for the LCLS rf photoinjector, Nucl. Instrum. Methods Phys. Res., Sect. A 429, 324 (1999).

[23] H. Qian et al., S-band photoinjector investigations by multiobjective genetic optimizer, in Proceedings of International Particle Accelerator Conference (IPAC'16), Busan, Korea (2016), Vol. 7, pp. 3979-3982.

[24] M. Krasilnikov et al., Experimentally minimized beam emittance from an L-band photoinjector, Phys. Rev. ST Accel. Beams 15, 100701 (2012).

[25] F. Zhou, A. Brachmann, P. Emma, S. Gilevich, and Z. Huang, Impact of the spatial laser distribution on photocathode gun operation, Phys. Rev. ST Accel. Beams 15, 090701 (2012).

[26] Y. Du, L. Yan, J. Hua, Q. Du, Z. Zhang, R. Li, H. Qian, W. Huang, H. Chen, and C. Tang, Generation of first hard $\mathrm{x}$-ray pulse at tsinghua thomson scattering $\mathrm{x}$-ray source, Rev. Sci. Instrum. 84, 053301 (2013).

[27] H. Chen, L. Yan, Q. Tian, Z. Lin, D. Wang, C. Cheng, J. Shi, H. Zha, Y. Du, H. Chen et al., Commissioning the photoinjector of a gamma-ray light source, Phys. Rev. Accel. Beams 22, 053403 (2019). 
[28] S. Mitri, On the importance of electron beam brightness in high gain free electron lasers, Photonics 2, 317 (2015).

[29] S. G. Anderson and J. B. Rosenzweig, Nonequilibrium transverse motion and emittance growth in ultrarelativistic space-charge dominated beams, Phys. Rev. ST Accel. Beams 3, 094201 (2000).

[30] X. He, C. Tang, W. Huang, and Y. Lin, A first-order analytical investigation on emittance evolution of relativistic space-charge dominated beams, Phys. Rev. ST Accel. Beams 560, 197 (2006).

[31] K. Floettmann, Emittance compensation in split photoinjectors, Phys. Rev. Accel. Beams 20, 013401 (2017).

[32] K. Floettmann, Astra: A space charge tracking algorithm.

[33] O. Luiten, S. Van der Geer, M. De Loos, F. Kiewiet, and M. Van Der Wiel, How to Realize Uniform ThreeDimensional Ellipsoidal Electron Bunches, Phys. Rev. Lett. 93, 094802 (2004).

[34] F. Zhou, A. Brachmann, P. Emma, S. Gilevich, and Z. Huang, Experimental studies with spatial gaussian-cut laser for the LCLS photocathode gun, in Proceedings of 33rd International Free Electron Laser Conference FEL 2011 (2011), pp. 341-344.

[35] H. Busch, Berechnung der Bahn von Kathodenstrahlen in axial symmetrischen electromagnetischen Felde, Ann. Phys. (N.Y.) 386, 974 (1926).

[36] V. Kumar, D. Phadte, and C. Patidar, A simple formula for emittance growth due to spherical aberration in a solenoid lens, in Proceedings of the DAE-BRNS Indian particle accelerator conference (2011).

[37] Z. Huang, Y. Ding, and J. Qiang, Analysis of slice transverse emittance evolution in a photocathode rf gun, Nucl. Instrum. Methods Phys. Res., Sect. A 593, 148 (2008).

[38] A. Mizuno, K. Masuda, and M. Yamamoto, Reduction of transverse emittance in electron injectors caused by space charge effects, Nucl. Instrum. Methods Phys. Res., Sect. A 774, 51 (2015).

[39] G. S. Gevorkyan, S. Karkare, S. Emamian, I. V. Bazarov, and H. A. Padmore, Effects of physical and chemical surface roughness on the brightness of electron beams from photocathodes, Phys. Rev. Accel. Beams 21, 093401 (2018).

[40] D. H. Dowell, F. Zhou, and J. Schmerge, Exact cancellation of emittance growth due to coupled transverse dynamics in solenoids and rf couplers, Phys. Rev. Accel. Beams 21, 010101 (2018).

[41] L. Zheng, J. Shao, Y. Du, J. G. Power, E. E. Wisniewski, W. Liu, C. E. Whiteford, M. Conde, S. Doran, C. Jing, C. Tang, and W. Gai, Experimental demonstration of the correction of coupled-transverse-dynamics aberration in an rf photoinjector, Phys. Rev. Accel. Beams 22, 072805 (2019). 